\title{
Análisis de las características de las incubadoras de empresas en Colombia: un estudio de casos
}

\section{Analysis of Characteristics of Business Incubators in Colombia: A Case Study}

\author{
Jesús C. Peña-Vinces ${ }^{1}$ \\ Saulo Bravo ${ }^{2}$ \\ Félix A. Álvarez ${ }^{3}$ \\ Duván A. Pineda ${ }^{4}$
}

Recibido: 11 de agosto, 2010

Aceptado: 28 de octubre, 2010

\section{Resumen}

Sobre la base de la metodología de investigación del estudio de casos, este artículo analiza las principales características que condicionan la supervivencia empresarial de cuatro incubadoras de empresas en Colombia (Bucaramanga, Créame, ParqueSoft y Gestando). Para ello se utiliza como instrumento la guía de buenas prácticas de las incubadoras de empresas de "Nodriza" en el análisis exploratorio. Los resultados revelan que las empresas cumplen en un 70\% con la guía de Nodriza, lo que les permite seguir sobreviviendo en el mercado nacional e internacional.

Palabras clave: Incubadora de empresas, pilares de supervivencia empresarial, redes empresariales, desarrollo regional, emprendedurismo.

\begin{abstract}
Based on the research methodology of case study, in this article we analyze the main characteristics that condition business's survival of four business's incubators in Colombia (Bucaramanga, Créame, ParqueSoft and Gestando). The researchers applied the guide of best practices of the business's incubators of Nodriza as a tool for the exploratory analysis. The results reveal that $70 \%$ of the companies comply with this guide, allowing them to continue surviving at the domestic and international market.
\end{abstract}

Key words: Business's incubators, pillars of business's survival, business networks, regional develop, entrepreneurship.

1. Ph. D. in Economics and Business (Universidad de Sevilla). Investigador y profesor de la Universidad de Sevilla, España <jesuspvinces@us.es>.

2. Ph. D. (c) in Business Adiministration (Universidad de Sevilla). Consultor empresarial y profesor investigador de la Universidad Santiago de Cali y de Intenalco.<sbravo@usc.edu.co>

3. Magíster en Ingeniería Industrial y de Sistemas (Universidad del Valle), Ingeniero Civil (Universidad del Valle). Consultor empresarial y profesor de la Universidad Santiago de Cali, Universidad Icesi y Universidad Javeriana.

4. Ingeniero Comercial (Universidad Santiago de Cali), Consultor de Empresas. 


\section{INTRODUCCIÓN}

La evolución y logros alcanzados por las incubadoras de empresas en Colombia han servido de soporte para el desarrollo socioeconómico de este país. Sin embargo, se han presentado situaciones en las que no se ha logrado alcanzar los objetivos, y una muestra clara de esto, es el alto porcentaje de empresas creadas dentro del proceso de incubación, que no han logrado sostenerse en el mercado. Según GEM (2005), la tasa de mortalidad de empresas en Colombia es una de las más altas de Latinoamérica (10.35\%), lo que indica que estos modelos de desarrollo empresarial aún requieren de ajustes para lograr un mejoramiento en el nivel de sostenibilidad de los emprendedores en los mercados, tanto locales como extranjeros (supervivencia empresarial).

Según los conceptos que se manejan en esta investigación, una incubadora de empresas se entiende como una entidad que acompaña a personas emprendedoras en la creación, impulso y consolidación de empresas preponderantemente innovadoras (Lalkaka, 2002). Esto implica ofrecer apoyos de capacitación en la elaboración de un plan de negocio y en ofrecer ambientes propicios para desarrollar el pensamiento empresarial. Lo antedicho requiere, además, vínculos con redes de apoyo tanto públicas como privadas, a través de diferentes alianzas estratégicas (Hu, Liu \& Qiu, 2009; López, Vallejo, \& González, 2009).

Esta investigación, mediante un estudio de casos, analiza las principales características - quizás las más importantes- de la supervivencia empresarial de cuatro incubadoras de empresas en Colombia: Bucaramanga, Créame, ParqueSoft y Gestando. Para ello utiliza la guía de buenas prácticas de incubadoras de empresas de Nodriza (2005). El desarrollo de este artículo se encuentra estructurado de la siguiente manera: en el primer apartado se hace una breve revisión de la literatura sobre las incubadoras de empresas que nos permitirá comprender el fenómeno de estudio. El siguiente apartado está dedicado a la muestra y metodología; es decir, aquí se detalla el marco teórico en que este trabajo se ha desarrollado, así como también los instrumentos de medida utilizados para el análisis de información cualitativa y que luego pudiera tener carácter cuantitativo. Con ello se quiere establecer un análisis comparativo para identificar cuál sería la incubadora que mejor cumple con los criterios de la guía Nodriza. En el penúltimo apartado, se presenta un resumen de los principales resultados obtenidos mediante el trabajo de campo. Finalmente, en la última sección se presentan las principales conclusiones, así como reflexiones e implicaciones para la gestión y la política.

\section{MARCO TEÓRICO}

Las incubadoras de empresas actualmente se han convertido en una reciente línea de investigación por la importancia que tienen para el desarrollo económico de los países. Sus inicios, según diversos estudios, datan a partir de la década de los años 70 del siglo pasado, preponderantemente en los Estados Unidos de Norteamérica y Europa Occidental.

La literatura revisada resalta la importancia que el tema va tomando como contribución al desarrollo socioeconómico y regional, mediante la creación y el fortalecimiento de empresas (Bravo, 2004; Hu, Liu \& Qiu, 2009; Lalkaka, 2002). Al mismo tiempo también resalta el esfuerzo de muchos investigadores por profundizar en este tema (Schwartz, \& Hornych, 2008; Walsh, Crockett \& Sheikholeslami, 2008; Yu, \& Nijkamp, 2009, y otros). Tal es así que en las incubadoras de empresas lo que se pretende es estimular a los emprendedores con el fin de dotar a sus ideas con las técnicas apropiadas para la creación de empresas sostenibles.

Las incubadoras pueden describirse como entidades impulsoras de conocimiento e investigación, ciencia y tecnología, fuentes generadoras de redes interinstitucionales, de estrategias de sostenimiento en el tiempo, de especialización en sectores determinados, de clasificación de incubadoras, la relación de inversionistas ángeles, la formalización de negocios informales, y la agrupación de empresas en unidades de negocios por sectores (Hu, Liu \& Qiu, 2009; Lalkaka, 2002). Pero las incubadoras no solo son desarrolladoras de conocimiento e investigación, también constituyen modelos 
de desarrollo económico (Nodriza, 2005). Tal es así que este nuevo tipo de empresas ayudan a fortalecer los talentos empresariales basados en la investigación y la innovación, diseñando estrategias de investigación desarrollo, y de comercialización de los productos generados en las empresas incubadas (Bravo, 2004). Por otro lado, Lalkaka (2003) argumenta que una incubadora de empresas es una infraestructura limitada con profesionales, servicios e instalaciones, ayudas técnicas y comerciales.

Una incubadora también es, pues, un programa que tiene por objetivo facilitar el surgimiento de emprendimientos o negocios de base tradicional y/o tecnológica. Ella brinda asistencia para que las nuevas empresas sobrevivan y crezcan durante su etapa de despegue, en la cual son más vulnerables (Hu, Liu \& Qiu, 2009). En general, las incubadoras ofrecen por tiempo limitado un espacio físico compartido con otras empresas; una vez vencido este, las empresas graduadas pueden optar por una nueva localización e independizarse de la incubadora. Un ejemplo claro de esto se puede encontrar en las incubadoras ubicadas en el parque científico y tecnológico de Sevilla (España), o en los parques biotecnológicos de Shanghái.

De este modo las incubadoras cumplen el papel de empresas matriz, ya que abrigan las ideas de los emprendedores y se enfocan en darle forma, desde sus inicios, invirtiendo principalmente en los estudios necesarios para la transferencia y la comercialización de tecnologías (Bravo, 2004; Hu, Liu \& Qiu, 2009; Lalkaka, 2002). Además, estas incubadoras ofrecen los recursos necesarios para alcanzar el objetivo propuesto, el cual debe de ser el de convertirse con el tiempo en una empresa fuerte y sólida, con una base tecnológica iniciada a partir de la idea primigenia. Al mismo tiempo se convierten en impulsoras de desarrollo socioeconómico de la localidad donde operan, permitiendo así que las empresas nacientes se desarrollen, participen y se mantengan activamente en un buen nivel competitivo. No obstante, es una labor que se lleva a cabo por un período límite. Aquí los emprendedores deben tener presente el largo plazo. En efecto, los emprendedores participantes deberán sensibilizarse y tomar conciencia de que hay que desarrollar altos niveles de eficiencia para poder participar en los mercados globales.

En definitiva, el éxito o supervivencia de una incubadora depende del aprovechamiento eficiente de sus recursos y capacidades para competir con éxito con otras empresas. En este sentido, las incubadoras de éxito prestan mucha atención al cumplimiento de criterios claves, como la buena gestión de personas, la gestión de sus recursos financieros, el desarrollo de redes empresariales, el desempeño organizacional, etc. (ver Figura 1) (Nodriza, 2005).

En Colombia, donde se realizó esta investigación, el $85 \%$ de las empresas que han pasado por incubadoras sigue funcionando. Según el promedio mundial, cerca del $70 \%$ de las empresas nuevas fracasan; sin embargo, la tasa de fracaso de las empresas que pasan por una incubadora se reduce de entre el 10 y el 15\% (Nodriza, 2005). Se estima que seis años es el tiempo promedio para la devolución de la inversión realizada por las empresas incubadas, vía pago de impuestos, creación de empleo y aportes parafiscales (Bravo, 2004). Al finalizar el año 2006 un total de 342 nuevas empresas colombianas de diferentes áreas productivas fueron creadas, gracias a la gestión de las 22 incubadoras de empresas de base tecnológica asociadas al proyecto Servicio Nacional de Aprendizaje de Colombia (SENA) (Bravo, 2004). Entre ellas generaron 1,871 empleos y reportaron un total de $\$ 21,514$ millones de pesos en ventas. A través de la cofinanciación de proyectos del SENA con recursos provenientes del programa nacional de apoyo y fortalecimiento de incubadoras de empresas de base tecnológica perteneciente al Departamento Administrativo de Ciencia y Tecnología e Innovación de Colombia (COLCIENCIAS), se han beneficiado un total de 108 proyectos presentados por emprendedores a través de las diferentes incubadoras asociadas entre los años 1999 y 2003, alcanzando así un monto total de colocación de \$10’245,662.116 (pesos colombianos). Desde 1999 hasta el 2006, el trabajo de las incubadoras de empresas asociadas al SENA se ve reflejado en la constitución de 982 nuevas empresas que generan 7,738 nuevos empleos y ventas por $\$ 173,154$ millones de pesos. En resumen, la razón de ser de las incubadoras 
de empresas es, sin lugar a dudas, sus incubados y sus propuestas de negocios. Y, sobre todo, la creación de empresas sostenibles en el tiempo.

\section{METODOLOGÍA Y MUESTRA DE ESTUDIO}

Para estudiar las características de las incubadoras de empresas en Colombia, se ha utilizado el estudio del “caso exploratorio" (Yin, 1994). La aplicación de esta metodología de investigación basada en la observación y la recogida de datos resulta imprescindible en las primeras etapas de una investigación, ya que permite contrastar y describir situaciones o hechos concretos (Cepeda, 2003). Las metodologías basadas en casos son habituales en investigaciones exploratorias sobre todo en entornos organizacionales complejos, $\mathrm{y}$ generalmente son empleados por investigadores de Administración de Empresas (Business Administration) (Cepeda, 2003; Marshall, 1985). Marshall y Rossman (1989) confirman la validez, lo apropiado y el valor de los métodos cualitativos, o estudios de casos, para los siguientes tipos de investigaciones: aquellos que examinan en profundidad cuestiones complejas y procesos; estudios sobre fenómenos poco conocidos o sistemas innovadores; análisis de las discrepancias entre principios y costumbres y comportamientos reales y conocimientos; estudios sobre relaciones poco estructuradas e informales y procesos en las organizaciones; investigaciones sobre objetivos organizativos reales (como opuestos a formales); trabajos en los que no se puede experimentar por cuestiones prácticas o éticas; e investigaciones sobre variables importantes que acaban de ser identificadas.

En este sentido, con el propósito de llevar a cabo el proceso de evaluación, se han estudiado las características más relevantes de las incubadoras más representativas de Colombia: Gestando, Créame, ParqueSoft, y Bucaramanga. Dichas incubadoras están ubicadas en las ciudades de Bogotá, Medellín, Cali y Bucaramanga respectivamente. En el trabajo se han estudiado los siete pilares básicos que se deben tener en cuenta en una incubadora para su supervivencia empresarial:
1) la gestión administrativa;
2) la gestión financiera;
3) la gestión del talento humano;
4) las infraestructuras;
5) el modelo de incubación;
6) las redes (networks); y,
7) la evaluación del desempeño.

Para realizar esta tarea se ha empleado la guía de buenas prácticas de las incubadoras de empresas de Nodriza. En definitiva, el estudio de caso realizado se construye sobre la evaluación de cada uno de los siete pilares propuestos por Nodriza. Donde se ha evaluado el cumplimiento o no de estos apartados. Para ello se ha utilizado la información contenida en cada una de las páginas web de las incubadoras nombradas. Por otro lado, se hizo necesario utilizar información adicional en algunos casos. Se empleó una escala dicotómica donde $1=$ Cumple y $0=$ No cumple con cada criterio.

Para determinar el nivel de cumplimiento de las prácticas de Nodriza era necesario contar con información cualitativa que luego pudiera tener carácter cuantitativo, con el fin de poder extraer, conclusiones y reflexiones. En este sentido, primeramente se sumaron cada una de las características que contiene Nodriza para cada empresa (ver Figura 1). Como criterio de evaluación general se tuvo en cuenta el total de características favorables identificadas en cada uno de los pilares de evaluación, para determinar porcentualmente si la incubadora realiza más o mejores acciones frente a las demás en el proceso emprendedor (ver Figura 2). Después de haber encontrado y seleccionado la información, se procedió a realizar el conteo de cada una de las características que cumple (identificadas), o no cumple (no identificadas) en cada incubadora. La cuantificación del cumplimiento se realizó teniendo en cuenta el número de características favorables sobre el número total de características evaluadas (Características Favorables /Total de Características*100). La cuantificación de incumplimiento se realizó teniendo en cuenta el número de características desfavorables sobre el total de características evaluadas (Características Desfavorables/ Total Características $* 100)$. 
Pilares de Supervivencia

Empresarial de las Incubadoras de Empresas

\section{Gestión administrativa}

- Plan estratégico (misión, visión, objetivos, políticas, metas y estrategias)

- Estructura de procesos de agrupación (clúster)

- Manual de incubadora

- Uso de medios masivos para promoción

- Estudios de mercado

- Mantenimiento de archivos detallados programas de referidos, seminarios y talleres

- Club de empresarios (fidelización)

- Publicación sobre desarrollos científicos

\section{Desempeño de las incubadoras}

- Indicadores de medición de las acciones del incubado

- Sistema de información de gestión que permita recolectar estadísticas

- Recolección de información anualmente para evaluar a los clientes

- Mecanismos de evaluación de las empresas

- Sistema de información para evaluación periódica

- Base de datos nacional de expertos como instrumento de evaluación de las ideas de negocio
Gestión del talento humano

- Perfil del director

- Capacitación continua de la dirección y demás miembros de la incubadora

- Gerentes expertos que apoyan los procesos de los incubados

- Programas de apoyo con voluntarios expertosy profesionales

- Evaluación continua de la gerencia a los incubados

- Manual de funciones

- Personal multidisciplinario y voluntarios generales (estudiantes, comunidad e industriales con experiencia)

\section{Infraestructura}

- Escenarios comunes

- Áreas de producción comunes como laboratorios

- Áreas de almacenamiento

- Comunicaciones y redes (LAN, banda ancha, etc.)

- Seguridad y sistemas eléctricos

- Localización estratégica frente a los stakeholders (académicos, bancos, etc.)

- Configuración del espacio

\section{Modelo de incubación}

- Portafolio de servicios

- Procesos de Coaching

- Mecanismos de participación y acuerdos de derechos de autor

- Implementación de incubadoras virtuales

- Laboratorios de ideas y un HELP DESK

- Personalidades universitarias en la asesoría especializada

- Centro de recursos legales

- Centro de competencia y formación

- Bolsa de trabajo

- Tasa variable sobre el canon de arrendamiento

- Apoyo gerencial

- Entrenamiento y facilitación de servicios y consejo de asesores

- Soporte para el desarrollo de la comercialización

- Filtros para seleccionar a los emprendedores y empresas que desean vincularse a la incubadora

- Contratos con los incubados

- Políticas de graduación

- Tiempo óptimo de incubación

- Criterios de selección

- Normas y procedimientos de admisión

- Sistema de seguimiento y monitoreo

- Programas de capacitación con universidades y colegios

- Programas de apoyo a la internalización de empresas incubadas

- Gestión de recursos para la internacionalización y globalización de losincubados

\section{Networking}

- Vínculos con universidades e instituciones de investigación

- Red de especialistas colaboradores

- Pool de mentores y directorios de consejeros con la adecuada experiencia y conocimiento

- Red de proveedores de servicios especializados pasantes/practicantes y empleados, etc.

- Vínculos con otros programas de incubación y emprendimiento

- Asociaciones industriales y organizaciones internacionales para compartir experiencias

- Publicaciones de investigación

- Asociación de emprendedores e innovación

- Eventos regulares y de promoción

- Redes de negocio y clúster

- Alianzas con profesionales de rubro tecnológico

\section{Gestión financiera}

\section{SOCIOS}

- Ingresos por arrendamientos y servicios (ingresos operacionales)

- Servicios a personas externas a los miembros (otros ingresos)

- Acuerdos con instituciones académicas

- Voluntarios que apoyan operaciones de la incubadora (pasantes, practicantes, etc.)

- Participación económica y accionaria en algún proyecto o empresa incubada

- Donaciones recibidas

- Fondos realizados por líderes empresariales conocidos

- Apoyos estatales

- Renta de otros espacios físicos de la incubadora

- Fondos provenientes de entidades de fomento (ONUDI, BID, INFODEV, CAF, etc)

- Aportes de empresas graduadas

- Subsidios

- Acuerdos de investigación de desarrollo

\section{CLIENTES}

- Capitales de riesgo

- Inversionistas ángeles

- Grupos de inversionistas

- Programas de crédito de organizaciones comunales y del gobierno

- Préstamos para capacitación de personal

- Programas de subsidio

- Instrumentos de fomento y fondos concursales

- Capital semilla

- Capital de acciones ordinarias

- Capital de deuda

- Fondos internos de capital

- Socios corporativos

Fuente: Elaboración propia con información de Nodriza (2005).

Figura 1. Los 7 pilares de análisis de las incubadoras de empresas 


\section{RESULTADOS DE LA INVESTIGACIÓN EXPLORATORIA}

\section{Incubadora Bucaramanga}

En pilar de la gestión administrativa, la información cualitativa nos revela que la corporación Bucaramanga Emprendedora (Bucaramanga) tiene un plan estratégico, a diferencia de las características de comercialización, de las cuales no se tiene información por ser primaria, además de la estructuración de procesos y su certificación. En otro de los aspectos relacionados con el marketing, a modo de ejemplo, esta empresa utiliza medios masivos de comunicación para su promoción como el Internet, la radio, la prensa y conferencias a gremios. Además, realiza estudios de mercado y competitividad a microempresas de diferentes sectores y diversas actividades de marketing, lo que garantiza a la corporación aumentar el impacto comercial frente al apoyo empresarial de otros emprendedores.

En cuanto a su financiación y sostenibilidad (pilar de gestión financiera), el estudio contempla tanto las características del ingreso como los acuerdos de cooperación y los vínculos con las distintas universidades de la región. Entre estas entidades, la incubadora coopera con la Universidad Industrial de Santander, la Universidad Autónoma de Bucaramanga, la Universidad Pontificia Bolivariana, Colciencias, y Sena. En el sector privado trabaja con Coomultrasan, Penagos Hermanos, Corporación Financiera Santander, Cámara de Comercio de Bucaramanga, Fundación Corona,
Fundeus, Metrogás, Gasoriente, Hugo Estrada Nieto y la Corporación Innova. En este pilar también se incluyen los recursos obtenidos por el arrendamiento de espacios físicos de la incubadora y los apoyos generados por el gobierno de Colombia; en este rubro se encuentran los acuerdos con la Gobernación de Santander y la Alcaldía de Bucaramanga.

Otra característica que tiene que ver con la financiación y sostenibilidad son los acuerdos de investigación $\mathrm{y}$ desarrollo, y financiación bancaria, los voluntarios que apoyan operaciones de la incubadora (pasantes, practicantes, etc.), y los acuerdos con instituciones académicas. En lo concerniente con la capitalización y financiación procedente de clientes, se observa que la incubadora cumple con las características tales como el capital de riesgo y programas de crédito, sustentados por el servicio de acceso a fuentes de financiación de la innovación, gracias a los acuerdos obtenidos con los entes territoriales, por la suscripción de la incubadora al Sistema Nacional de Información Educativa de Colombia (SNIE) y al Fondo Emprender del Servicio Nacional de Aprendizaje de Colombia (SENA).

La incubadora cumple con cada una de las características que exige la evaluación del pilar de la gestión del talento humano, concretamente en la dirección y estructura organizacional. No obstante, su gerencia y staff no cumplen totalmente con las características de evaluación. Ello se debe a la carencia de información primaria, sobre todo en la característica denominada perfil del director. La Tabla 1 resume cuantitativamente

Tabla 1.

Resultados del Análisis Exploratorio de Bucaramanga Emprendedora

\begin{tabular}{lcc}
\hline \multicolumn{1}{c}{ Pilares de Evaluación } & Cumplimiento (\%) & No Cumplimiento (\%) \\
\hline Gestión Administrativa & 91 & 9 \\
Gestión Financiera & 70 & 30 \\
Gestión del Talento Humano & 1 & 99 \\
Infraestructura & 71 & 29 \\
Modelo de Incubación & 96 & 4 \\
Networks & 91 & 9 \\
Evaluación e Impacto & 71 & 29 \\
\hline Fuente: Elaboración propia. & &
\end{tabular}

J. econ. finance adm. sci., 16(30), 2011 
los resultados de la evaluación de los siete pilares de la incubadora Bucaramanga.

Siguiendo con el análisis de los resultados, la capacitación continua de la dirección y demás miembros de la incubadora Bucaramanga, gerentes expertos que apoyan en los procesos de los incubados y programas de apoyo con voluntarios expertos y profesionales, se interpretó bajo el supuesto de que las acciones descritas en los diferentes medios de divulgación se están realizando en los programas de apoyo con voluntarios expertos y profesionales. Se aplicó el concepto de cumplido, gracias a los acuerdos de cooperación establecidos con las diferentes instituciones de educación superior de la región.

Por otro lado, y con la finalidad de comprender mejor las diferentes prácticas y la relación entre incubadoraincubados, el proceso de selección y graduación de incubados contiene las características determinantes de este proceso. No obstante, una buena relación, así como un proceso adecuado y serio, permiten que se creen nuevos negocios con un alto nivel de éxito empresarial. En el caso específico de Bucaramanga, se podría interpretar de manera positiva el logro mayoritario en cada una de las características. Por ejemplo, aquí se trabaja con el establecimiento de un portafolio de servicios para sus incubados, la definición de procesos de coaching que acompañan el proceso del emprendedor, la fijación de mecanismos de participación, y acuerdos de derechos de autor y la implementación de incubadoras virtuales, entre otras. También se incluye el apoyo gerencial, el entrenamiento y facilitación de servicios, consejo de asesores y soporte para el desarrollo de la comercialización.

En el caso del pilar de la infraestructura física, esta incubadora brinda espacios comunes, áreas de producción, tales como laboratorios, áreas de almacenamiento, comunicaciones y redes (LAN, banda ancha, etc.), seguridad, sistemas eléctricos, localización estratégica frente a los stakeholders (académicos, bancos, etc.), y configuración del espacio. Además, cumple con todas las características del pilar, teniendo en cuenta la descripción que hace la incubadora y las evidencias fotográficas de las instalaciones físicas. Por ejemplo, se advierte el uso de despachos comunes para los incubados, áreas de producción comunes, y el uso mancomunado de las tecnologías de la información y comunicación.

Sobre el pilar de las redes y vínculos, así como sus relaciones con el entorno, se puede decir que en esta incubadora las variables han sido factores que han permitido mejorar las relaciones con los distintos grupos sociales o stakeholders del incubado; es decir, las redes tanto humanas como de organizaciones permiten dar soporte y sostenibilidad a las nuevas ideas de negocios; primero, sopesando la incertidumbre que genera el futuro, y segundo, prevaleciendo las acciones del proceso emprendedor que asume el incubado. En este caso se puede concluir que esta cuenta con una red que se adecúa a las necesidades del emprendedor, desde la relación con el gobierno, entidades del sector educativo, el sector privado y financiero y, por supuesto, su red primaria compuesta por proveedores, clientes y otros incubados.

En el último pilar de evaluación, la medición e impacto de las incubadoras, se cuenta con las estadísticas e indicadores de la gestión que estas realizan para medir el nivel de competitividad y para analizar sí se están cumpliendo los objetivos previstos por la incubadora. Bucaramanga Emprendedora tiene un sistema de información de gestión que le permite recolectar estadísticas de los emprendedores, de las empresas vinculadas y de su clasificación o estado de vinculación (pre-incubación, incubación o post incubación), así como los aportes que sus asociadas hacen. Otras características inherentes son la recolección de información anual para evaluar el programa de la incubadora y a los clientes, así como el sistema de información para la evaluación periódica. Todo esto se realiza gracias al uso de las tecnologías de información como herramienta de soporte y control. Sin embargo, Bucaramanga Emprendedora no cumple con algunas de las características debido al bajo nivel de información virtual, en cuanto a los indicadores de medición de las acciones del incubado y mecanismos de evaluación de las empresas incubadas.

De acuerdo con la evaluación general, Bucaramanga Emprendedora logró identificarse con el 85\% de las acciones que la entidad realiza correspondientes a gestión administrativa, gestión financiera, infraestructura, 
modelo de incubación, redes y evaluación e impacto (ver Tabla 1). Entre ellos se perciben picos importantes donde la incubadora presenta una buena evaluación.Tal es el caso, por ejemplo, de modelo de incubación con un cumplimiento del $96 \%$ de las actividades requeridas para la evaluación. Le sigue la gestión administrativa con $91 \%$ y la gran red de la que dispone Bucaramanga (91\%). Los grupos de infraestructura y evaluación e impacto llegan a $71 \%$, de representatividad frente a estos factores.

Sin embargo, algunas de las explicaciones hacen referencia a la carencia de indicadores de medición de las acciones del incubado y de mecanismos de evaluación de las empresas como herramientas de control, de la evaluación e impacto del proceso. Por otro lado, en cuanto a infraestructura, se desconocen algunas características como las áreas de almacenamiento y la configuración de espacios. Un aspecto preocupante de esta incubadora se relaciona con la gestión de sus recursos humanos que solo ha logrado obtener una puntuación del 1\%.

\section{Incubadora Créame}

En el pilar de gestión administrativa, la información reveló que Créame ha estado desarrollando una serie de actividades administrativas que le han permitido poseer una clara visión de futuro y una buena retroalimentación de su entorno. En cuanto a la planificación, se ha identificado que la incubadora posee un alto nivel de estructuración y organización. La gerencia juega un papel muy importante en el proceso de planeación y orientación, frente a su estructura institucional. En cuanto estrategias de marketing, Créame realiza un buen trabajo frente a cada una de las actividades de marketing, sobre todo en el uso de Internet, la radio y medios de prensa escrita. En este último rubro, Créame utiliza canales de comunicación masivos para la interacción tanto de sus incubados como con los demás grupos de interés, así como la interacción e intercambio de información con los emprendedores a través de encuestas y test de evaluaciones. También programa y realiza eventos empresariales, y difunde publicaciones de investigación.
En lo referido al pilar de financiación, Créame incluye otros subgrupos, tales como soporte, financiación y sostenibilidad, y capitalización y financiación de clientes. De acuerdo con la información disponible, se puede afirmar que esta incubadora cumple con el $63 \%$ del total de las características de evaluación (ver Tabla 2). Entre algunos aspectos destacados se pueden distinguir la tenencia de convenios y acuerdos con distintas universidades de la región (Universidades de Antioquia, Medellín, Nacional de Colombia, etc.). Asimismo, dentro de su estructura corporativa, se puede destacar la participación de la Gobernación de Antioquia y la Alcaldía de Medellín, así como otras entidades académicas y privadas que apoyan y financian la corporación, además de los diferentes acuerdos de investigación y desarrollo que mantienen con el sector educativo de la ciudad y la región.

Sobre la capitalización y financiación de clientes, y según las características más relevantes, Créame cumple satisfactoriamente con las acciones de gestión de consecución de capitales de participación, como por ejemplo capitales de riesgo, inversionistas ángeles y grupos inversores, realizadas por la empresa Capitalia Colombia. En el caso de la financiación de los proyectos de incubados vía créditos, se encontró que existen socios corporativos financieros, capitales semilla, además de los programas de créditos tradicionales realizados y facilitados por los bancos asociados. Sin embargo, se ignora si existen fondos internos de capital, programas de subsidios, capital de acciones ordinarias, y otros.

Por otro lado, en Créame se destaca la capacitación permanente de los asociados y demás miembros de la incubadora, así como los programas de apoyo de voluntarios y profesionales, técnicos, expertos, etc. Se debe considerar la firma de convenios y acuerdos de cooperación con las diferentes organizaciones de carácter académico. Este aspecto es propio de las incubadoras de empresas, especialmente de las denominadas intramuros, caracterizadas por ofrecer a los emprendedores en la fase de inicio espacios adecuados en los que se incluyen los servicios de secretaria, puestos de trabajo, acceso a Internet, particularmente necesarios en esta etapa de iniciación. 
Aunque Créame ofrece servicios de asesoría y acompañamiento, no se sabe si existen infraestructuras para los emprendedores que deseen obtener un espacio dentro de la incubadora. Se asume que se cuentan con algunos espacios específicos como el dedicado a las capacitaciones de los incubados, el uso de tecnologías de comunicaciones y redes, seguridad y sistemas eléctricos entre otros. La incubadora también cuenta con un amplio portafolio de servicios, un proceso definido de coaching, un centro de recursos legales a través de su aliado A\&G Auditoria y Gestión, además de un centro de competencia, formación y desarrollo comercial, a través de servicio de outsourcing comercial.

Otro aspecto relevante es que la incubadora ha establecido un proceso para la selección y aprobación de proyectos empresariales exitosos. Este proceso se caracteriza por sus filtros de selección, criterios de selección, normas y procedimientos, contratos de incubación, políticas de graduación, tiempo de incubación, además de otras características. Por último, Créame apoya el proceso de internacionalización de cada una de sus empresas con potencial exportador a través de sus aliados internacionales, como por ejemplo Euroffice Services y Makaia.

Una de las acciones más importantes es la constitución de una gran red de apoyo y cooperación (pilar de las redes). La red de Créame está formada por las Universidades de la región, el sector empresarial, los gobiernos locales, la banca y grupos de interés, entre otros actores. También cuenta con un proceso de réplica del modelo a través de nodos de apoyos establecidos en las principales ciudades del país, entre ellas Cafetero, Medellín, Cali, Cesar, Arauca y Barranquilla.

En términos relativos a la organización, Créame tiene una estructura de evaluación de cada uno de sus procesos. Ya que esta estructura de evaluación es un mecanismo interno, la información obtenida es secundaria. Algunas de las características que se pudo obtener, aunque correspondiente a lo identificado, fueron las siguientes: la incubadora realiza el control a todo el proceso del incubado para conocer su estado; asimismo, aplica tecnologías de la información como herramienta de control y evaluación para obtener información estadística del estado de los incubados, del total de empresas, de los recursos generados y otros aspectos.

Entre los rangos poco favorables para Créame, se pudo identificar que los grupos de gestión financiera e infraestructura no cuentan con características identificables que contribuyan a mejorar la función o consecución de los objetivos propuestos por la entidad. En el caso de gestión financiera (pilar de gestión financiera), Créame obtuvo $63 \%$, donde la consecución de recursos para la sostenibilidad son reducidos de acuerdo con la gestión desarrollada por la entidad. En infraestructura obtuvo el $57 \%$ ya que no cuenta con espacios de almacenamiento, configuración del espacio y la localización frente a los diferentes grupos de interés. Sobre este aspecto, la Tabla 2 resume las puntuaciones obtenidas en cada uno de los pilares de la evaluación de la incubadora Créame.

Tabla 2.

Resultados del Análisis Exploratorio de Créame

\begin{tabular}{lcc}
\hline \multicolumn{1}{c}{ Pilares de Evaluación } & Cumplimiento (\%) & No Cumplimiento (\%) \\
\hline Gestión Administrativa & 91 & 9 \\
Gestión Financiera & 63 & 37 \\
Gestión del Talento Humano & 90 & 10 \\
Infraestructura & 57 & 43 \\
Modelo de Incubación & 78 & 22 \\
Networks & 100 & 0 \\
Evaluación e Impacto & 100 & 0 \\
\hline Fuente: Elaboración propia & &
\end{tabular}

J. econ. finance adm. sci., 16(30), 2011 


\section{Incubadora ParqueSoft}

En lo referente al enfoque y planificación (pilar de gestión administrativa), ParqueSoft cuenta con planes claros frente a su futuro a corto y mediano plazo; es decir, cuenta con un plan estratégico bien definido con el conocimiento como columna vertebral de soporte para la supervivencia empresarial. También entiende que su estructura se fundamenta en la integración horizontal, entre cada uno de sus miembros y mediante la formación del clústeres tecnológicos de la región. ParqueSoft trabaja con clústeres por la facilidad de transferencia de conocimientos, lo que difícilmente se puede lograr sin la integración. Es posible, entonces, encontrarse clústeres especializados en el desarrollo de software.

En las actividades de marketing, ParqueSoft realiza diferentes actividades de promoción y comunicación efectiva para persuadir a diversos grupos sociales de interés. Para tal efecto utiliza la Internet como medio tecnológico masivo de comunicación; también promueve la participación en eventos empresariales, educativos y otros, así como la participación en talleres y ferias de tecnología.

Con respecto al pilar referido a la financiación, ParqueSoft garantiza su sostenibilidad y sustentabilidad a través de la consecución de capital y demás recursos económicos. Una de las características más relevantes de la organización es que ParqueSoft dispone de un modelo compartido de gasto, valorado por metro cuadrado, donde cada empresario aporta mensualmente para sufragar los servicios de Internet, servicios públicos, alquiler de instalaciones, etc. Además, cuenta con fuentes de trabajo permanente mediante convenios con instituciones de educación superior de la región, obteniendo así recursos por la oferta de servicios tecnológicos a clientes e incubados. Otras de las acciones que le permiten a ParqueSoft obtener recursos es a través de apoyos estatales realizando eventos internacionales, en conjunto como, por ejemplo, con la gobernación del Valle del Cauca, el Ministerio del Medio Ambiente de Colombia, y el Instituto de Promoción de las Exportaciones Colombianas (PROEXPORT). Estas actividades las ejecuta a través de su departamento de proyectos, el que se encarga de presentar diferentes propuestas ante fondos provenientes de entidades de fomento, tales como ONUDI, BID, INFODEV, CAF, PNUD, el BID EE.UU, la UE, entre otros. También, busca firmar acuerdos de investigación y desarrollo financiados mediante créditos bancarios.

Con referencia a la capitalización y financiación de los clientes, ParqueSoft ha desarrollado estrategias y alianzas con los diferentes sectores financieros, con el propósito de gestionar recursos para los nuevos proyectos y sus empresas incubadas. Además, la incubadora se asocia con otras organizaciones en joint ventures de capital, coaliciones para la financiación de proyectos a través de capitales de riesgo, como de inversionistas ángeles del sector empresarial, fondos internos de capital, capital semilla y fondos concursales. Otras acciones que realiza sobre este tema son la gestión de créditos ordinarios para las empresas incubadas, la financiación de programas de capacitación a empleados e incorporación de socios corporativos para la capitalización de sus empresas incubadas.

En lo referido al pilar de la gestión del talento humano, ParqueSoft es una organización con buenos cimientos institucionales; cuenta con un personal idóneo y altamente calificado. Se debe destacar el liderazgo del equipo gerencial en el cumplimiento de las metas y objetivos propuestos, basado en unas políticas coherentes con los intereses de la incubadora y sus incubados. Entre algunas de las actividades más importantes realizadas por la dirección de la organización es el compromiso por desarrollar programas de capacitación continua de los incubados y demás miembros de la incubadora. Aquí tenemos, por ejemplo, el programa HumanWare donde estudiantes universitarios, profesores, e incubados se capacitan con expertos en computación y tecnologías de la información, y también se forman semilleros para la investigación y el emprendimiento. Asimismo, la dirección del ParqueSoft cuenta con un personal multidisciplinario y con experiencia en los diferentes temas, además de la evaluación constante a los incubados.

Por otro lado, sobre infraestructura tecnológica ParqueSoft ha centrado sus esfuerzos para crear una 
infraestructura tecnológica de clase mundial, infraestructura que le ha permitido a los emprendedores desarrollar soluciones de gran calibre, y a los que ya han despegado, generar negocios. Para ello, provee a todas las empresas de diversos apoyos logísticos adecuados para el desarrollo de sus negocios. Algunos de estos apoyos son las denominadas salas de negocios. Estas salas cuentan con ayudas visuales como sistemas de comunicación, red de alta velocidad de Internet, así como áreas de producción comunes como los laboratorios computacionales, dotados con software desarrollador, aplicativo, ofimática, entre otros. La ubicación estratégica también le permite a ParqueSoft poseer la visión de desarrollar un polo tecnológico al sur de la ciudad de Cali, lugar que aglutina a la mayoría de las universidades de la región.

En el pilar de modelo incubación, ParqueSoft cuenta con un amplio portafolio de servicios que comprende la asesoría personalizada de expertos en computación y tecnologías de la información, y el acompañamiento, o coaching, hasta la ejecución y puesta en marcha de nuevas empresas con un alto componente de conocimiento aplicado. Otra de las características de ParqueSoft es que cuenta con infraestructura de laboratorios, un modelo de gasto compartido en el caso de arrendamiento, un centro de competencia y formación. Por medio de su plan HumanWare, se promueven semilleros de investigación, apoyo gerencial y alto soporte para el desarrollo de la comercialización de los productos de los incubados, utilizando técnicas en inteligencia de negocios, desarrollo de negocios, y mercadeo creativo, entre otras acciones.
Estas acciones son un ejemplo de un polo generador de desarrollo empresarial continuo.

Dentro de este mismo apartado, en lo relacionado con la selección y graduación de incubados, los resultados revelaron que esta incubadora cuenta con un riguroso proceso de filtración de ideas de negocios de los emprendedores, al ser una organización con un alto nivel de empresas innovadoras y con propuestas tecnológicas. Dicho proceso se realiza con una planilla de inscripción en donde se presenta el proyecto; posteriormente se hace una cita con el Comité de Emprendimiento para determinar si es coherente con la filosofía y los criterios de la incubadora. A diferencia de los modelos tradicionales de incubación, ParqueSoft no mantiene un tiempo definido para salir del modelo, es decir, una empresa entra y sale cuando lo desee. En el caso del apoyo a internacionalización de los incubados, ParqueSoft promueve programas de internacionalización con sus aliados estratégicos, bajo el modelo de desarrollo de negocios; al mismo tiempo, gestiona los recursos económicos de apoyo que ofrece PROEXPORT.

En el pilar de las redes, todos los modelos asociativos en ParqueSoft han formado redes de apoyos con otras organizaciones, así como actores que acompañen y compartan el proceso. Algunas de estas organizaciones son las universidades del Valle, San Buenaventura, Autónoma, Javeriana, Icesi, y Santiago de Cali. Otras alianzas estratégicas para la investigación y transferencia de tecnología se han formado con MICROSOFT, IBM, ORACLE (quienes proveen software de desarrollo),

Tabla 3.

Resultados del Análisis Exploratorio de ParqueSoft

\begin{tabular}{lcc}
\hline Pilares de Evaluación & Cumplimiento \% & No Cumplimiento \% \\
\hline Gestión Administrativa & 91 & 9 \\
Gestión Financiera & 89 & 11 \\
Gestión del Talento Humano & 90 & 10 \\
Infraestructura & 83 & 17 \\
Modelo de Incubación & 78 & 22 \\
Networks & 91 & 9 \\
Evaluación e Impacto & 86 & 14 \\
\hline Fuente: Elaboración propia. & &
\end{tabular}

J. econ. finance adm. sci., 16(30), 2011 
y los centros de producción de software libre, como FREEWARE y OPEN SOURCE. También se incentiva la participación constante de investigadores expertos en computación y tecnologías de la información, así como la alianza con ICONTEC para la certificación de los productos en ISO-8999, entre otras acciones.

En el último pilar de análisis de evaluación e impacto, en términos generales se puede decir que la organización tiene una estructura de evaluación de cada uno de sus procesos. Ya que este es un mecanismo interno, no se puede obtener información de mayor profundidad, aunque aparentemente presenta un manejo adecuado de datos estadísticos con sistemas de información, y el uso de las tecnologías de información. En la Tabla 3 se resume las puntuaciones obtenidas en cada uno de los pilares de evaluación de acuerdo con la guía Nodriza.

\section{Incubadora Gestando}

$\mathrm{Al}$ realizar una revisión general de las características de la incubadora Gestando Empresarial Colombia, se detectaron vacíos de información o información insuficiente y no relevante para esta investigación. Del 96\% de las características seleccionadas, el 51\% aproximadamente no cumple o se desconoce de la información.

En el caso del pilar de gestión administrativa, la incubadora Gestando cuenta con un plan estratégico. La empresa pertenece a un clúster claramente identificado que agrupa a diferentes empresas de su sector, especialmente en la promoción de empresas llamadas de economía social. Gestando se crea en palabras de Antonio Salcedo, gerente de la incubadora, para "establecer un modelo de incubadoras en el sector solidario es una de las características más importantes a resaltar del proceso de incubación". El cambio de concepto de préstamo a los incubados, de los tradicionales créditos de consumo, por créditos productivos ha permitido que los asociados emprendedores desarrollen actividades empresariales innovadoras y exitosas. Sin embargo, una de las mayores desventajas identificadas en esta incubadora es su débil posicionamiento como cooperativa incubadora de empresas; esta podría ser una de las razones por las cuales en el subgrupo denominado marketing no cuenta con un índice favorable sobre la información encontrada (ver Tabla 4). Esto significa que la incubadora deberá trabajar mejor sus acciones de mercadeo para promocionarse masivamente.

En el pilar de gestión financiera, la incubadora cuenta en general con un nivel de calificación aceptable acorde con la guía Nodriza, especialmente en cuanto a la búsqueda de recursos para el mantenimiento, financiación y sostenibilidad de las acciones que realiza la incubadora. Una de las características más importantes en la obtención de recursos son los aportes de sus asociados, además de los ingresos percibidos por el servicio de incubación a los emprendedores y demás servicios profesionales. Algunas de las acciones para reducir el gasto son los convenios de cooperación desarrollados con la incubadora Créame. Con relación a la financiación de los proyectos de sus incubados, la institución gestiona convenientemente sus recursos mediante capitales de riesgo, inversionistas ángeles, grupos de inversionistas, programas de crédito de organizaciones comunales y del gobierno colombiano, capital semilla y la adquisición de préstamos por parte de sus asociados.

El pilar de gestión del talento humano es sin lugar a dudas una de las características más interesantes que muestra esta incubadora. Según se estudian los datos de la Tabla 4, resalta la estructura jurídica que posee la organización; es un modelo de desarrollo solidario como cooperativa, que promueve y apoya la creación de nuevas empresas a costes asequibles para sus incubados.

Otras de las características resaltantes de la incubadora son sus programas de soporte, los cuales realiza particularmente con expertos y profesionales, quienes apoyan a los incubados desde la formulación de planes de negocio, hasta la puesta en marcha de su idea. Además, aquí se ve la importancia de contar con un personal multidisciplinario e idóneo que acompañe en el proceso a los incubados.

El pilar de infraestructura es uno de los temas del que no se tiene información alguna de esta incubadora. Aunque establece dentro de sus procesos el concepto de extramuros, esto no es más que el apoyo a distancia de 
la entidad al emprendedor desde la etapa de iniciación hasta la puesta en marcha de su empresa. Esto indica el requerimiento de infraestructura para el desarrollo como son el arrendamiento, disposición de equipos de comunicación, redes locales, equipos de oficina, y tecnologías de la información operando mediante el sistema de intramuros.

En el pilar de análisis sobre el modelo de incubación, la incubadora cuenta con un portafolio de servicios para ofertar a los emprendedores. Entre estos se distinguen el apoyo frente a la formulación de planes de negocio, el permanente acompañamiento de personal capacitado mediante un proceso de coaching, programas de formación en competencias empresariales e iniciación en emprendimiento y temas de gestión de empresas. Otra de las características fundamentales que se destaca es el respaldo de la gerencia frente a las acciones de los emprendedores, además del apoyo ofrecido por medio de un departamento dedicado a proyectos especiales. Esta área se encarga de comercializar el conocimiento generado por los convenios establecidos entre las incubadoras (Créame-Gestando).

De otro lado, Gestando también se caracteriza por la selección y graduación de los incubados. Con relación a lo primero, la incubadora realiza dos convocatorias para recibir ideas de negocios que son filtradas con el fin de seleccionar las mejores propuestas. El tiempo de graduación del incubado dura alrededor de los tres y seis meses con un coste de $\$ 600,000$ pesos colombianos para el emprendedor.

Otra característica destacable en Gestando es en el pilar de redes con la formación de programas de capacitación con universidades y colegios, en donde se promueven semilleros empresariales para jóvenes estudiantes de colegios y universitarios. De este rubro o grupo, el aspecto característico de la incubadora es el convenio realizado con Créame y con otros agentes económicos donde se establecen prioridades de cooperación en temas de proyectos de desarrollo tecnológico.

El último pilar de análisis es la evaluación e impacto. En este grupo la única información que presenta son estadísticas en cuanto al número de empresas creadas con el apoyo de la incubadora. Según se indica, hasta el presente son 40 las empresas que han generado nuevos empleos.

En resumen, en el caso de Gestando se puede decir que la gestión del talento humano es el grupo más relevante del que se conoce información. Gestando aparece con un $70 \%$ de cumplimiento seguido del modelo de incubación con un $52 \%$, la gestión administrativa $45 \%$, la gestión financiera $48 \%$, y evaluación e impacto con un $43 \%$. Se debe señalar que los pilares que obtienen menor puntuación serían el pilar de infraestructura y redes con un porcentaje menor al 50\% (ver Tabla 4).

Tabla 4.

Resultados del Análisis Exploratorio de Gestando

\begin{tabular}{lcc}
\hline \multicolumn{1}{c}{ Pilares de Evaluación } & Cumplimiento (\%) & No Cumplimiento (\%) \\
\hline Gestión Administrativa & 45 & 55 \\
Gestión Financiera & 48 & 52 \\
Gestión del Talento Humano & 70 & 30 \\
Infraestructura & 29 & 71 \\
Modelo de Incubación & 52 & 48 \\
Networks & 27 & 73 \\
Evaluación e Impacto & 43 & 57 \\
\hline
\end{tabular}

Fuente: Elaboración propia. 


\section{ESTUDIO COMPARATIVO DE LAS INCUBADORAS DE EMPRESAS DE COLOMBIA}

Después de haber efectuado el estudio de cada una de las incubadoras, en cada uno de los siete pilares de supervivencia empresarial y en función de la data disponible, se elaboró un modelo de análisis comparativo con el fin de determinar cuál sería la incubadora que cumple con mayor amplitud los criterios de Nodriza. En otras palabras, ¿cuál sería la incubadora con mayor éxito empresarial con relación al resto de incubadoras? La Figura 2 presenta las cuatro incubadoras de empresas aquí estudiadas.

De acuerdo con el modelo del radar de la evaluación de las incubadoras de empresas, se puede decir que en primer lugar la incubadora ParqueSoft es la empresa que cumple ampliamente con los 7 pilares de evaluación tal como se puede observar en la Figura 2, exceptuando el pilar de gestión administrativa. En segundo lugar se observa a Créame, cuya única dificultad es en lo que respecta a las redes empresariales. En tercer lugar se ubica la incubadora Bucaramanga Emprendedora. En ella destacamos los serios problemas de su gestión en recursos humanos. Por su parte, la incubadora Gestando sería la empresa que cumpliría con menos criterios de la guía de las buenas prácticas de Nodriza, ya que su radar de evaluación es el más pequeño de todos comparándolo entre las cuatro incubadoras. En resumen, los puntos en el radar en que en la incubadora que se encuentra más alejada del límite de evaluación (extremos del radar) indican que esos son pilares más débiles y que, por lo tanto, deberá mejorar en ellos, si no quiere poner en riesgo su supervivencia empresarial y la de sus incubados.

\section{CONCLUSIONES E IMPLICACIONES PARA LA GESTIÓN y LA POLÍTICA}

Como conclusión general se puede afirmar que, de las cuatro incubadoras aquí analizadas, en tres de ellas se observa un desempeño promedio por encima del $75 \%$. La cuarta incubadora (Gestando) se encuentra por debajo del 50\% de cumplimiento de la guía de las buenas prácticas de Nodriza. Ello significa que la supervivencia empresarial de esta última estaría en peligro en el largo plazo. De lo anterior puede afirmarse que, en términos generales, el desempeño empresarial o la supervivencia de las incubadoras de Colombia es notable.

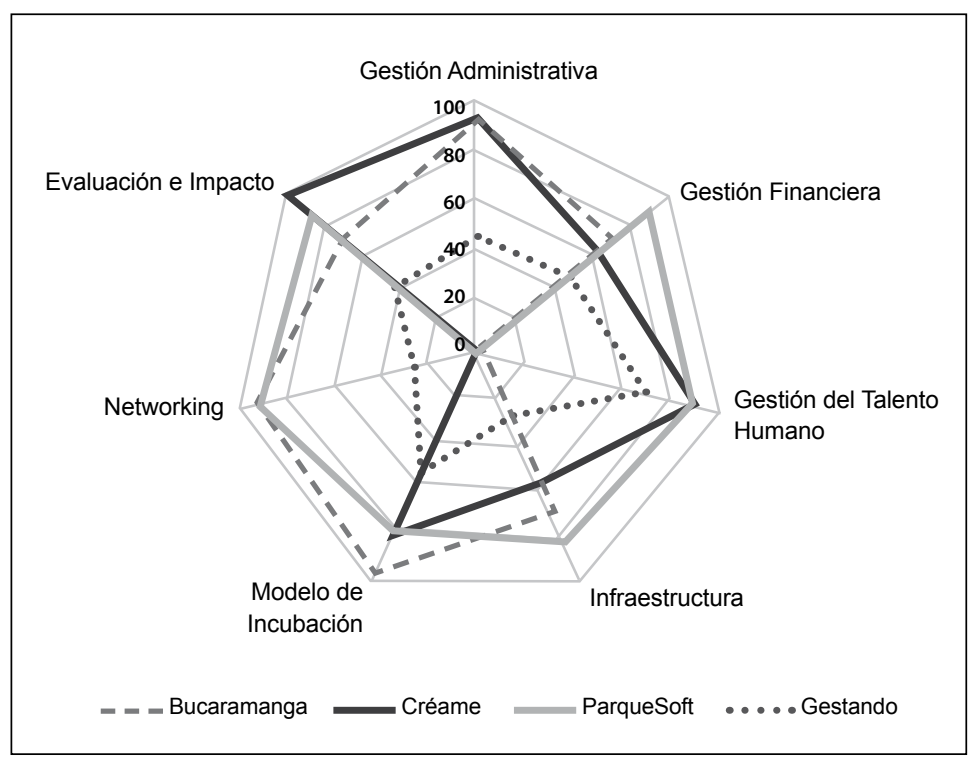

Figura 2. Modelo comparativo del radar de la evaluación de las incubadoras de empresas de Colombia. 
Como se ha demostrado en este estudio, tal y como sucede con otro tipo de empresas, en las incubadoras es igualmente importante la aceptación de las organizaciones ante sus stakeholders. En este sentido, a las incubadoras y a sus incubados no solo les interesa la venta de sus productos/servicios, sino que también les interesa la relación con clientes y empleados. De igual forma, el número de redes de negocios y redes sociales con las que cuentan las incubadoras también ayudan a la supervivencia de este nuevo modelo de organización empresarial. Asimismo, debe reconocerse la importancia del empleo de Internet como medio principal de comunicación masiva entre las incubadoras y sus incubados para la transferencia e intercambio de información.

Al mismo tiempo, es indudable la necesidad de la capacitación constante y el coaching como factores fundamentales que también han ayudado a la supervivencia de los incubados. De acuerdo con el enfoque de las incubadoras (Incubators Based View, Lalkaka, 2002; 2003), no se puede dejar solos a los polluelos (incubados) hasta que estos no sean capaces de poder volar por si solos. En otras palabras, se deberá evitar poner en riesgo la supervivencia de los proyectos incubados hasta su éxito final. Como se ha visto en el caso de las cuatro incubadoras analizadas, todas en ellas acompañan a sus incubados hasta que estos son capaces de competir y rivalizar por sí solos en mercados globalizados.

Nuestra investigación también ha demostrado que las incubadoras de empresas son generadoras de desarrollo económico de las regiones y países. Gracias a los acuerdos que estas organizaciones mantienen con las universidades, sus egresados cuentan con un empleo, o generan su autoempleo, durante o al finalizar su formación. En muchos casos, además, logran convertirse en pequeños empresarios y, como afirma Peña-Vinces (2009), son las empresas tanto privadas como públicas las que generan el desarrollo económico de los países y no el país en sí mismo; en este caso en particular nos referirnos a las incubadoras de empresas.

Tales proyectos animan a los gobiernos a seguir apostando por este nuevo modelo de desarrollo económico basados en incubadoras de negocios. En efecto, las incubadoras son vistas como uno de los motores core (centrales) del desarrollo económico y social (Liu \& Qiu, 2009; Lalkaka, 2002) que vienen cumpliendo un papel muy relevante. En Colombia, operan comercialmente sobre todo en lugares donde el desempleo puede alcanzar fácilmente hasta el $64.7 \%$. En la solución de este problema macroeconómico, las incubadoras en particular se han convertido en los lugares preferidos de la gente que quiere trabajar independientemente. Aquí los emprendedores desarrollan sus ideas de negocios; en otras palabras, las incubadoras han elevado la cultura empresarial, como por ejemplo el desarrollo industrial, consiguiéndose de esta manera involucrar a los diversos niveles de gobiernos, tanto nacional como regional.

Por otro lado, las incubadoras están acortando la brecha que existe entre la universidad y las empresas, particularmente en Sudamérica. Como se ha podido ver en los resultados analizados en cada una de las incubadoras, estas mantienen convenios de cooperación con las principales universidades de la zona, para el desarrollo de proyectos conjuntos de I+D (Investigación y Desarrollo). En definitiva, las incubadoras de empresas en Colombia están cumpliendo con su objetivo principal: servir de plataformas de despegue para las personas que tienen una idea de negocios y que están dispuestas a desarrollarla. Del mismo modo, las incubadoras de empresas han ayudado en los procesos de internacionalización de muchas pequeñas empresas. En este sentido, consideramos importante señalar que aquellas empresas que aún no han entrado en la exportación lo hagan mediante este tipo de organizaciones, pues ellas son puentes de acceso a los mercados extranjeros.

De igual manera, los resultados del estudio muestran que las incubadoras son impulsoras de conocimiento e innovación, al mismo tiempo que permiten la consolidación de empresas de base de tecnológica. Esto es mucho más dramático en países en vías de desarrollo donde la tecnología es muy escasa y no está al alcance de cualquier empresa por sus elevados costes, su desarrollo e implementación (Peña-Vinces, Chin \& Cepeda, 2011). 
En este caso en particular, la incubadora ParqueSoft ha desempeñado un papel importantísimo al convertirse en una de las empresas líderes y con presencia internacional en el desarrollo de nuevas tecnologías. Pero, no solo han logrado la consolidación de empresas de base tecnológica, sino que también ha conseguido la especialización de sectores industriales. Por ejemplo, ese es le caso de Bucaramanga Emprendedora en biotecnología, gracias a la cooperación que consolidó con otros grupos empresariales.
Las infraestructuras físicas dentro de las instalaciones de las incubadoras, tales como laboratorios, oficinas comunes y tecnologías, cumplen un rol fundamental en el desarrollo empresarial de sus incubados. Tales establecimientos permiten que sus miembros formen nuevas redes, tanto empresariales como personales. Al mismo tiempo, los proyectos incubados consiguen su formalización legal lo que ha permitido que las empresas nacientes tengan acceso a la financiación bancaria gracias al paraguas de la incubadora matriz.

\section{Referencias}

Bravo, S. (2004). Perspectivas de un desarrollo económico local basado en un sistema de incubadora de empresas y redes sociales en Santiago de Cali, Valle del Cauca. Proyecto de tesis doctoral no publicado, Dpto. de Administración de Empresas y Marketing. Universidad de Sevilla-España

Centro Integral de Servicios Empresariales (Créame) (2005). Recuperado el 20 de diciembre de 2005 en $<$ www.creame.com.co $>$.

Cepeda, G. A. (2003). Gestión del conocimiento, capacidades diferenciales y ventaja competitiva: análisis de sus relaciones. Tesis doctoral, Dpto. de Administración de Empresas y Marketing, Universidad de Sevilla-España.

Corporación Bucaramanga Emprendedora (Bucaramanga) (2005). Recuperado el 20 de diciembre de 2005 en $<$ www.bucaincu.org $>$.

Departamento Administrativo de Ciencia y Tecnología e Innovación de Colombia (Colciencias) (2005). Decreto Ley 344 de 1996 financiación de proyectos para creación de empresas. Recuperado el 20 de diciembre de 2005 en <http://www.colciencias.gov.co/>.

Frenkela, A., Shefera, D., \& Millera, M. (2008). Public versus Private Technological Incubator Programmes: Privatizing the Technological Incubators in Israel. European Planning Studies, 16(2), 189-210.

Fundación Parque Tecnológico del Software (ParqueSoft) (2005). Recuperado el 20 de diciembre de 2005 en $<$ www.parquesoft.com>.
Global Entrepreneurship Monitor, (GEM) (2005). GEM Global Reports. Recuperado el 20 de diciembre de 2005 en <http://www.gemconsortium.org/about. aspx?page=pub_gem_global_reports $>$.

Hu, B., Liu, R., \& Qiu, Z. (2009). How to Enhance the Efficiency of University Student Incubator: the Application of Total Service Quality Management Model. Trabajo presentado en la Fourth International Conference on Computer Sciences and Convergence Information Technology. Seúl, Corea del Sur.

Lalkaka, R. (2002). Technology Business Incubators to Help Build an Innovation-based Economy. Journal of Change Management, 3(2), 167-176.

Lalkaka, R. (2003). Business Incubators in Developing Countries: Characteristics and Performance. International Journal of Entrepreneurship and Innovation Management, 3(1-2) 31-55

López, J., Vallejo, M., \& González O. (2009). Manual para la gestión en incubación del sistema nacional de creación e incubación de empresas de Colombia. Recuperado el 24 de enero de 2009 en $<$ http://www. icesi.edu.co/ciela/036.pdf>.

Marshall, C. (1985). Appropriate Criteria of Trustworthiness and Goodness for Qualitative Research on Education Organizations. Quality on Quantity, 19, 353-373.

Marshall, C., \& Rossman, G. (1989). Designing Qualitative Research. Newbury Park, CA: Sage. 
Nodriza Incubation Partner (Nodriza) (2005). Guía de buenas prácticas para las incubadoras de empresas, Recuperado el 20 de diciembre de 2005 en<http:// www.negociosyemprendimiento.org/2011/01/ buenas-practicas-incubadoras-empresas.html $>$.

Peña-Vinces, J. C. (2009). Comparative Analysis of Competitiveness on the Peruvian and Chilean Economies from a Global View. Journal of Economics, Finance and Administrative Science, 14(27), 87-105.

Peña-Vinces, J. C., Chin, W. W., \& Cepeda, G. A. (2011). The Effect of Use of the Information Technologies and Communications on Firms' International Performance and their Constraints in Development Countries: the Case of Peruvians' Multilatinas. Trabajo presentado en las XXI, Jornadas Hispano-Lusas de Investigación Cientifica Córdova, España.

Schwartz, M. \& Hornych, C. (2008). Specialization as Strategy for Business Incubators: an Assessment of the Central German Multimedia Center. Technovation, 28, 436-449.

Scillitoe J. N., \& Chakrabarti, A. K. (2010). The Role of Incubator Interactions in Assisting New Ventures. Technovation, 30(3) 155-167.
Servicio Nacional de Aprendizaje de Colombia (Sena) (2004). Recuperado el 20 julio de 2004 en $<\mathrm{http}: / /$ emprendimiento.sena.edu.co.mayo2004>.

Walsh, D., Crockett, R., \& Sheikholeslami, M. Z. (2008). Project Based Learning as a Catalyst for Academic Evolution and as an Incubator for Academic Innovation. Trabajo publicado en los Proceedings of the 38th Frontiers in Education Conference. Saratoga Springs, New York.

Yin, R. K. (1994). Case Study Research: Design and Methods (2nd Ed.). Thousand Oaks, CA: Sage Publications, Inc.

Yu, J., \& Nijkamp, P. (2009). Methodological Challenges and Institutional Barriers in the Use of Experimental Method for the Evaluation of Business Incubators: Lessons from the US, EU and China. Trabajo presentado en la Atlanta Conference on Science and Research Policy. Georgia, USA. 